\title{
Eating Behavior of Students at the Technical University of Moldova during the Isolation Period
}

\author{
Rodica Siminiuc ${ }^{1,2 *}$, Eugenia Covaliov ${ }^{1,2}$, Dinu Țurcanu ${ }^{2,3}$, Daniela Pojar ${ }^{2,4}$, \\ Vladislav Reșitca ${ }^{1,2}$, Aurica Chirsanova ${ }^{1,2}$, Tatiana Capcanari ${ }^{1,2}$ \\ ${ }^{1}$ Food and Nutrition Department, Technical University of Moldova, Chisinau, Republic of Moldova \\ ${ }^{2}$ Technical University of Moldova, Chisinau, Republic of Moldova \\ ${ }^{3}$ Informatization, Partnerships, Institutional Image and Communication Office, Chisinau, Republic of Moldova \\ ${ }^{4}$ Financial Affairs and International Relations Office, Chisinau, Republic of Moldova \\ Email: ^rodica.siminiuc@adm.utm.md, eugenia.boaghi@toap.utm.md, dinu.turcanu@adm.utm.md, \\ daniela.pojar@adm.utm.md,vladislav.resitca@adm.utm.md, aurica.chirsanova@toap.utm.md,tatiana.capcanari@toap.utm.md
}

How to cite this paper: Siminiuc, R., Covaliov, E., Țurcanu, D., Pojar, D., Reșitca, V., Chirsanova, A. and Capcanari, T. (2022) Eating Behavior of Students at the Technical University of Moldova during the Isolation Period. Food and Nutrition Sciences, 13, 108-123.

https://doi.org/10.4236/fns.2022.132011

Received: December 7, 2021

Accepted: February 6, 2022

Published: February 9, 2022

Copyright $\odot 2022$ by author(s) and Scientific Research Publishing Inc. This work is licensed under the Creative Commons Attribution International License (CC BY 4.0).

http://creativecommons.org/licenses/by/4.0/

\begin{abstract}
Appetizing peculiarities are formed from childhood and can vary throughout life. Although they are conditioned by genetic factors, over time, the internal regulation of eating behaviors is reduced, being influenced by a complex interaction of various external factors. According to the Behavioral Susceptibility (BST) Theory of Obesity, Food Sensitivity in Response to Food Stimuli (such as Food Sight and Smell) and Satiety Responsiveness (i.e. the ability to adjust diet in response to internal feelings of satiety) contribute to individual differences in terms of energy intake and weight status. The present research intended to assess the eating patterns and behavior of Moldovan students, especially those from Technical University of Moldova during the social isolation imposed by the COVID-19 pandemic. The study was conducted, based on the questionnaires (AEBQ-Adult Eating Behavior Questionnaire), on a group of 602 students from Technical University of Moldova. Participation in the survey was entirely voluntary, did not involve any invasive procedure, nor did it induce changes in participants' food patterns. The obtained results could contribute to the formation of the database for the study of the behavioral phenotype associated with the risk of obesity, but also for the study of eating behavior in crises. The questionnaire was distributed, in the form of a link, on the corporate emails of all TUM students. Respondents completed the questionnaire on the Google platform between 13.10.2021 - 25.11.2021, and the final database has been downloaded as a Microsoft Excel file. It was established a positive correlation between, Emotional Over-Eating and Emo-
\end{abstract}


tional Under-Eating. Nervousness and anger were the most incriminating emotions of students that would cause both overeating and under eating. The results of the AEBQ questionnaire appear to be largely in line with previous studies, and the collected data present interest, in particular to prevent the risk of obesity.

\section{Keywords}

Eating Behavior, Students, Emotional Eating, Hunger, Satiety

\section{Introduction}

Peculiarities of eating behavior are formed from childhood and can vary throughout life. Although it is known that the genetic factor conditions the risk of obesity, as we age, the internal regulation of eating behaviors decreases, being influenced by a complex interaction of different external factors [1] [2] [3]. Stress and anxiety also amplify each other's influence on emotional adjustment strategies and eating behaviors, increasing the risk of overeating in vulnerable people [4].

In addition to the high number of casualties, the COVID-19 pandemic is an unprecedented challenge, with profound social and economic consequences. As the chronology of the growing pandemic is uncertain, isolation has been exacerbated by panic, depression, insomnia, and mass anxiety [5] [6].

It has been shown that loss of normal routine and reduced social and physical contact with others often lead to boredom, frustration, and a sense of isolation from the rest of the world [7]. Chronic loneliness and boredom, if long enough, can have detrimental effects on physical and mental well-being, as well as eating habits and patterns [8]. Changes in food patterns have led to increased consumption of unhealthy foods [9] [10] [11] but also more time spent cooking [12]. Procurement of processed or semi-processed foods has been reduced by almost 50\% [10]. Traffic limits within and between countries affect food-related logistics services and lead to disruption of food supply chains, affecting food availability [13]. And dietary restrictions can paradoxically make individuals more susceptible to unhealthy eating when their ability to self-regulate is threatened. Previous experiments have shown that in situations that cause low self-control and/or unhealthy cravings, participants with higher food retention eat more than those with lower retention [8] [14] [15].

Public health nutrition experts warn us about the importance of a balanced diet and maintaining an active lifestyle to promote proper eating habits during the isolation period. Students, although considered young adults, are at a critical juncture in terms of food choices and behaviors [16], and the restrictions imposed by the pandemic may exacerbate these choices.

According to the Behavioral Susceptibility (BST) Theory of Obesity, Food Sensitivity in Response to Food Stimuli (such as Food Sight and Smell) and Sa- 
tiety Responsiveness (i.e. the ability to adjust diet in response to internal feelings of satiety) contribute to individual differences in terms of energy intake and weight status. Sensitivity to satiety, hunger, emotional eating, external responsiveness are aspects of appetite that have been shown to be associated with adiposity [1] [17].

Some researchers point out that the characteristics of the food approach (receptivity to food, emotional overeating and the pleasure of eating food) are positively correlated with patterns of overconsumption (more frequent consumption of food). On the other hand, receptivity to satiety, emotional sublimation, Food Fussiness, and Slowness in Eating are negatively correlated with the pattern of malnutrition [18]. At TUM, as at any other university, the restrictions imposed by the pandemic led to the realization of classes in online mode, as well as in hybrid mode [19] [20]. The consequences of the impact of isolation on physical and mental well-being, as well as eating habits and patterns, have not been explored.

Assessing the eating behavior of young TUM students would be a way to study the behavioral phenotype associated with the risk of obesity. And the strong link between nutrition and the immune system only increases the imperative of this study. At the same time, the study could help us to shape the reactions to the inevitable future pandemics [10].

\section{Materials and Methods}

Survey methodology. A study was conducted, based on the questionnaires, on a group of TUM students $(n=602)$. Participation in the survey was entirely voluntary, did not involve any invasive procedure, nor did it induce changes in participants' food patterns, nor did it require the approval of an ethics committee. Participants were informed about the purpose of the research. The questionnaire was distributed, in the form of a link, on the corporate emails of all TUM students. Respondents completed the questionnaire on the Google platform between 13.10.2021 - 25.11.2021, and the final database has been downloaded as a Microsoft Excel file.

Respondents. Of the 602 young adults aged $18-26,345$ are men and 257 are girls.

The questionnaire included 2 general items (gender and age) and 35 items related to eating behavior. Eating behavior was assessed according to the Adult Eating Behavior Questionnaire (AEBQ) [21]. The 35 items of AEBQ are to provide information on 8 aspects: Enjoyment of food, Emotional over-eating, Emotional under-eating, Food fussiness, Food responsiveness, Slowness in eating, Hunger, Satiety responsiveness(Appendix 1).Answers were provided on a Likert scale from 1 to 5 points: strongly disagree $(S D)=1$, disagree $(D)=2$, Neither agree or disagree $(\mathrm{NAD})=3$, agree $(\mathrm{A})=4$, strongly agree $(\mathrm{SA})=5$ (represented by the values $1-5$ on the $\mathrm{X}$ axes in Figures 1-8). In order to facilitate the reinterpretation of the data, only the answers with a score of 4 and 5 were taken into 
account, and the answers with a score of 1 and 2, as well as 3 (as a neutral answer) were omitted.

Data analysis. The results of the survey were analyzed by descriptive statistics. The Cronbach's alpha coefficient was calculated to determine the full reliability of the questionnaire (Table 1 ).

\section{Results and Discussions}

The answers to the 35 items were grouped into 8 groups, which in turn were divided by gender and represented in the form of diagrams.

Enjoyment of food. It is believed that the joy of eating should play an important role in our food consumption [22]. From the results of the survey, we notice that there are no big differences regarding the joy of food between genders: $76 \%$ of boys and $70 \%$ of girls love food, $76 \%$ - 77\% like to eat and only $35 \%$ of young people look forward to mealtime (Figure 1(M) \& Figure 1(F)).

Table 1. Descriptive statistics and Cronbach's alpha coefficient.

\begin{tabular}{|c|c|c|c|}
\hline \multirow{3}{*}{ Eating behavior } & \multicolumn{2}{|c|}{ Descriptives } & Alpha \\
\hline & $\mathbf{M}$ & SD & \multirow{2}{*}{0.84} \\
\hline & 2.92 & 1.15 & \\
\hline
\end{tabular}

M-means, SD—standard deviation, Alpha (Cronbach's alpha coefficient).

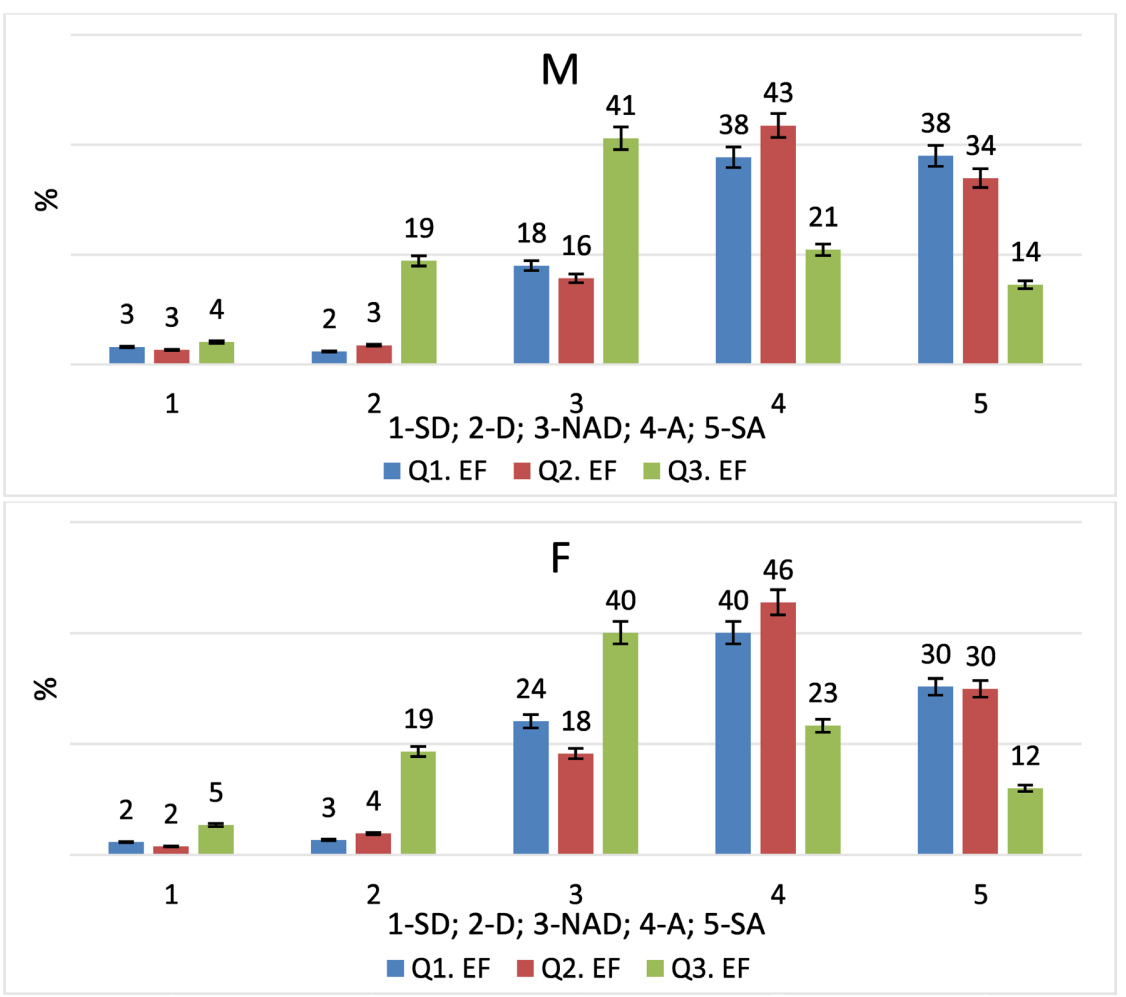

Figure 1. (M) Enjoyment of food. Q1. EF-I love food; Q2. EF-I enjoy eating, Q3. EF-I look forward to mealtimes, $\mathrm{M}-$ male gender. (F) Enjoyment of food. Q1. EF-I love food; Q2. EF-I enjoy eating, Q3. EF-I look forward to mealtimes, $\mathrm{F}-$ female gender. 
Emotional over-eating \& under-eating. Emotional overeating (EOE) has been associated with an increased risk of obesity, while emotional underfeeding (EUE) may be protective [23]. Research on adults has shown that some people tend to consume more in stressful situations (so-called emotional overeating, [EOE]), while others experience a decrease in appetite when stressed and eat less (so-called emotional overeating). emotional malnutrition, [EUE] [24]. Regarding our study, in general, there is a positive correlation between, EOE and EUE.

In the case of emotional over-eating, the results showed that most young people are not subjected to overeating due to emotions. The vulnerability in this aspect is higher for girls, compared to boys, at least during this pandemic period (when the survey was conducted). Thus, $21 \%$ of boys and $37 \%$ of girls eat more when they are annoyed, $18 \%(\mathrm{M})$ and $26 \%(\mathrm{~F})$-eat more when they are worried, 13\% (M) and 26\% (F)-when are upset, 8\% (M) and 24\% (F)-when they feel anxious and $12 \%(\mathrm{M})$ and $28 \%(\mathrm{~F})$ - when they are angry (Figure $2(\mathrm{M}) \&$ Figure 2(F)).

Emotional under-eating. There is a positive correlation between responses to emotional over-eating and emotional under-eating: a very high positive correlation for boys $(r=0.87)$ and a high correlation for girls $(r=0.78)$ (Figure 3(M) \&

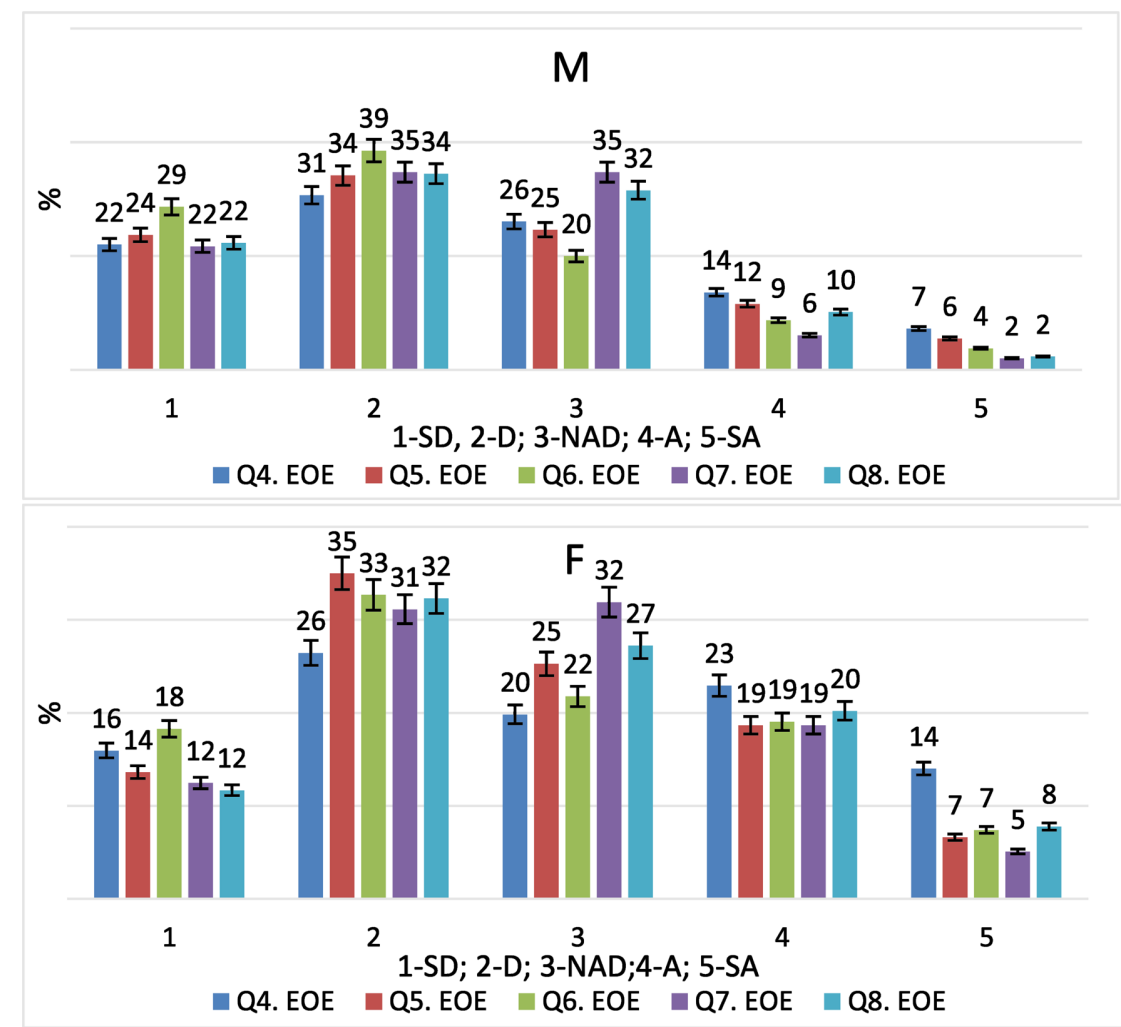

Figure 2. (M) Emotional over-eating. Q4. EOE-I eat more when $I$ am annoyed; $Q 5$. EOE-I eat more when I am worried; Q6. EOE-I eat more when I am upset, Q7. EOE-I eat more when $1 \mathrm{~m}$ anxious, Q8. EOE-I eat more when I am angry. (F) Emotional over-eating. Q4. EOE-I eat more when I am annoyed; Q5. EOE-I eat more when I am worried; Q6. EOE-I eat more when I am upset, Q7. EOE-I eat more when $I m$ anxious, Q8. EOE-I eat more when I am angry. 


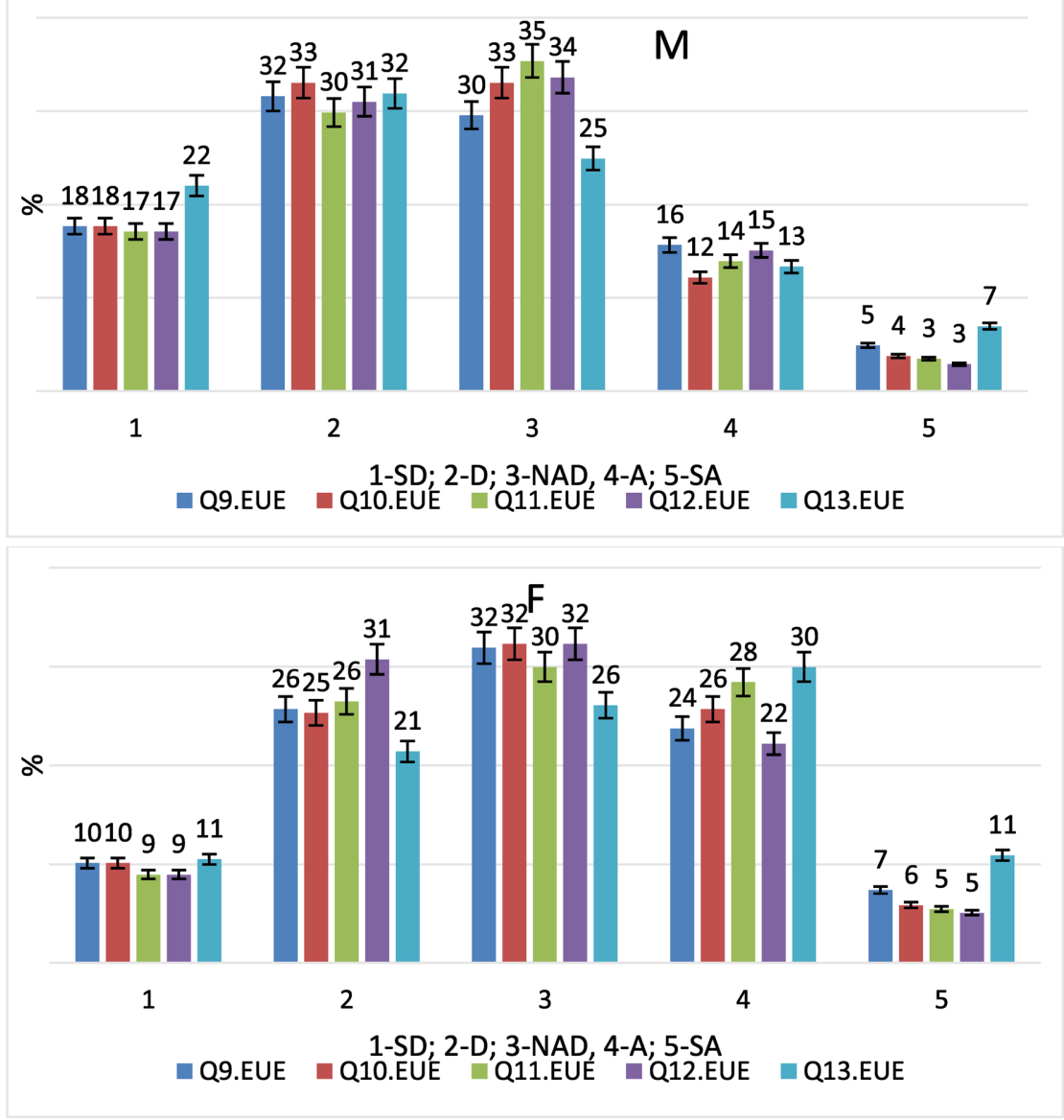

Figure 3. (M) Emotional under-eating. Q9. EUE-I eat less when I'm worried; Q10. EUE-I eat less when I'm angry; Q11. EUE-I eat less when I'm upset, Q12. EUE-I eat less when I'm annoyed; Q13. EUE-I eat less when I'm anxious. (F) Emotional under-eating. Q9. EUE-I eat less when I'm worried; Q10. EUE-I eat less when I'm angry, Q11. EUE-I eat less when I'm upset, Q12. EUE-I eat less when I'm annoyed; Q13. EUE-I eat less when I'm anxious.

Figure $3(\mathrm{~F})$ ). Emotional over-eating correlated positively with hunger $(\mathrm{r}=0.83$ (B) and $r=0.86(F)$, correlations confirmed by previous research on this topic) [25].

Food fussiness. Food fussiness is considered to be a concept related to both genetic and environmental etiologies. It is also thought to be associated with traits such as anxiety and shyness [26]. Boys (60\%) like to taste food more than girls (22\%). 59\% of young people are interested in tasting new foods and over $66 \%$ of young people like a wide variety of foods. Respondents (39\% (M) and $44 \%(F))$ often decide that they do not like a food even before consuming it. Only a small proportion of young people $(13 \%-14 \%)$ refuse to taste new foods (Figure 4(M) \& Figure 4(F)).

Food responsiveness. Appearance and olfactory sensations are the stimuli that most often lead to the desire to eat food. The relationships between taste receptivity and pleasure are dependent on the product, but also on the individual receptivity to basic tastes [27]. 


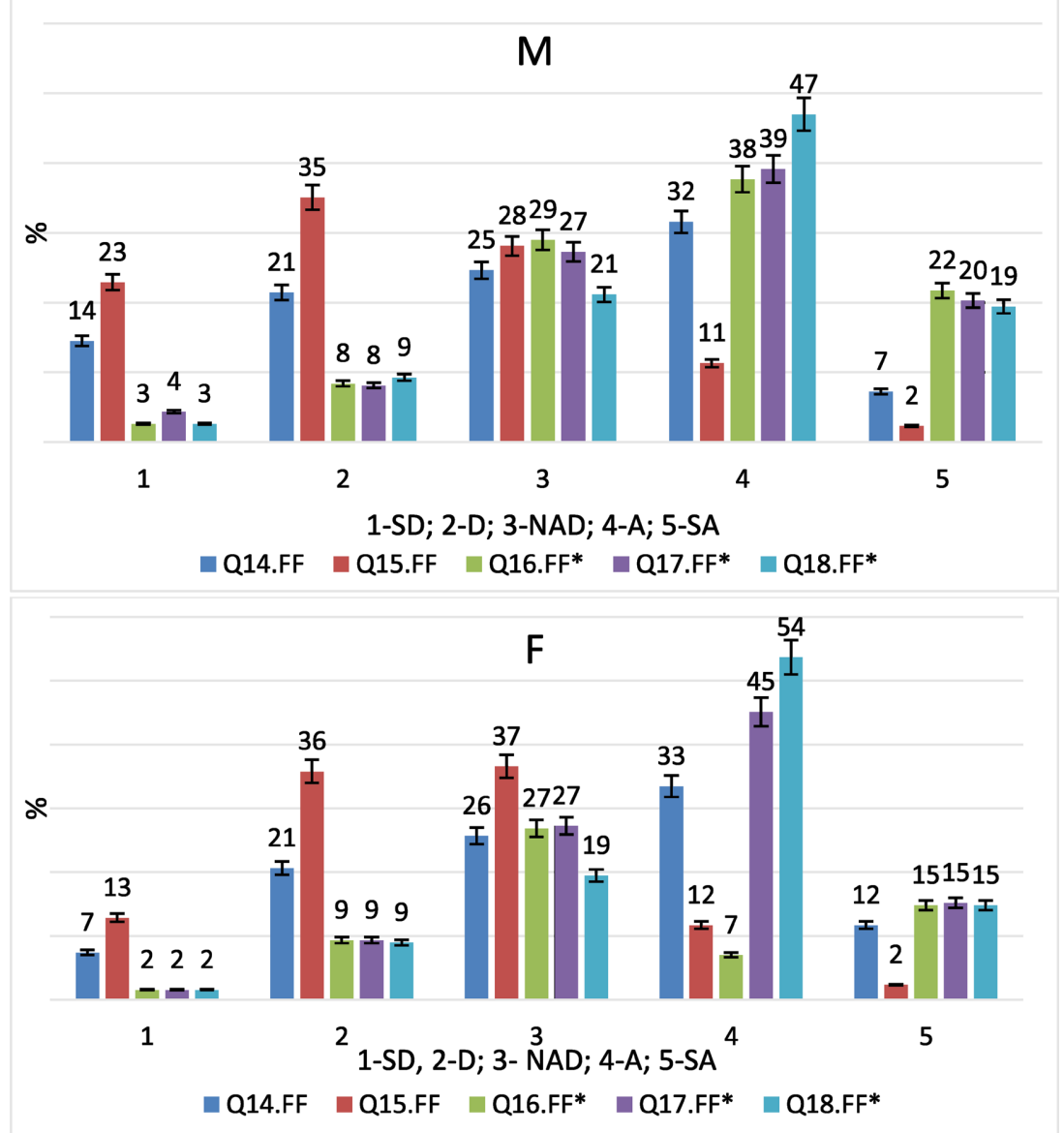

Figure 4. (M) Food fussiness. Q14. FF-I often decide that I don't like a food, before tasting it, Q15. FF -I refuse new foods at first, Q16. FF*-I enjoy tasting new foods, Q17.FF* $-I$ am interested in tasting new food I haven't tasted before, Q18. FF*-I enjoy a wide variety of foods. (F) Food fussiness. Q14. FF-I often decide that I don't like a food, before tasting it, Q15. FF-I refuse new foods at first, Q16. FF*-I enjoy tasting new foods, Q17. FF* $-I$ am interested in tasting new food I haven't tasted before, Q18. FF* $-I$ enjoy a wide variety of foods.

Over $41 \%$ of respondents want to eat when they are in the presence of someone who eats, and $23 \%$ of boys and $13 \%$ of girls would most often prefer to eat. Appearance and olfactory sensations are the stimuli that most often lead to the desire to eat food-over $61 \%$ of respondents confirmed this fact (Figure 5(M) \& Figure 5(F)).

According to BST theory, body weight is influenced, at least in part, by genetic factors, through their effect on appetite. Schather reported that obese people have two instinctive characteristics in regulating their appetite that lead to overeating: excessive receptivity to very tasty foods (wanting to eat a lot at sight, smell and taste of tasty foods) and lack of response to internal mechanisms of satiety [2] [28].

Slowness in eating. There is research that shows that eating a small amount of food slowly and eating a large amount of food quickly are just as effective in causing satiety, and the rate of eating does not affect the feeling of hunger and 


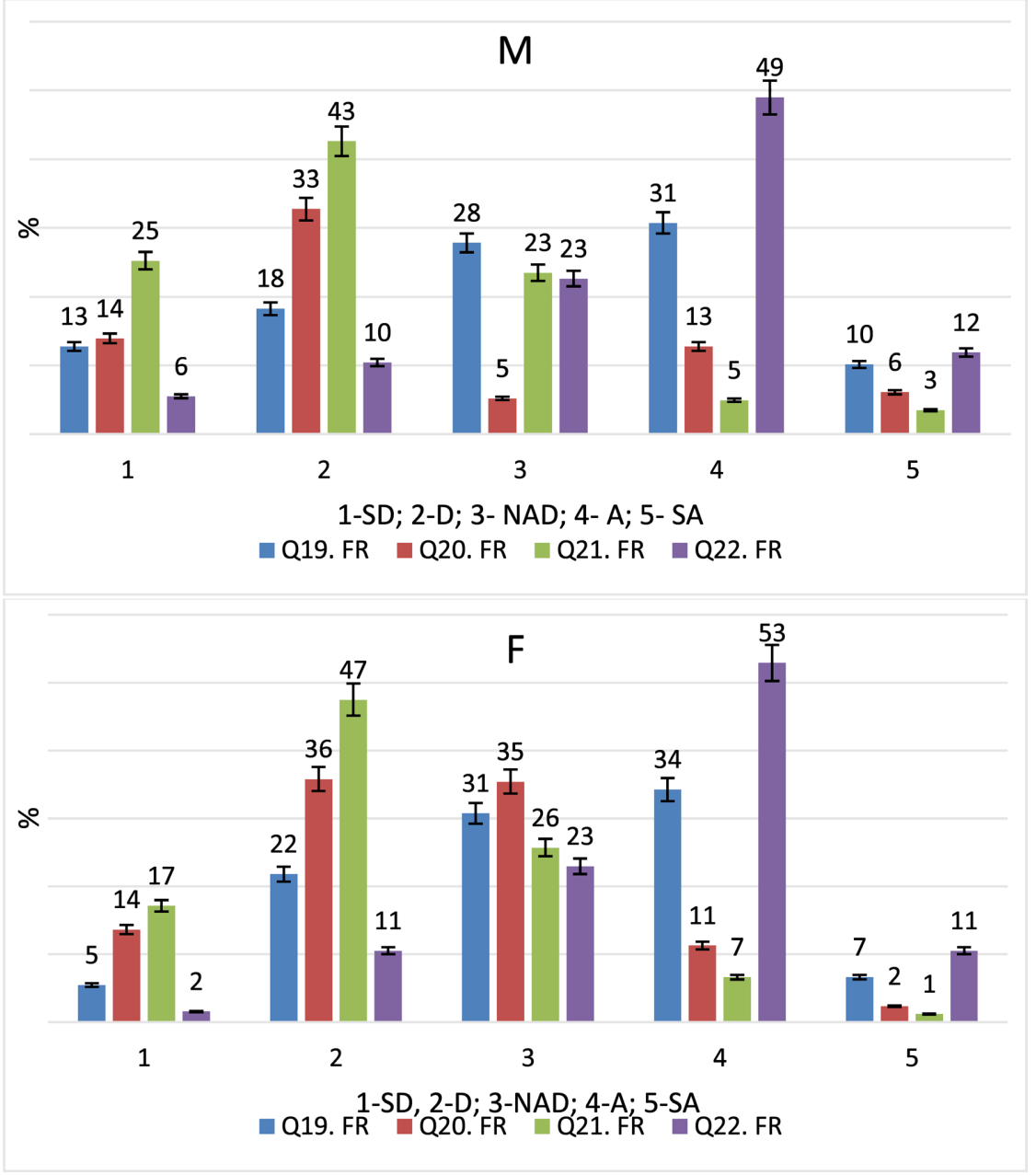

Figure 5. (M) Food responsiveness. Q19. FR-I often feel hungry when I am with someone who is eating, Q20. FR-Given the choice, I would eat most of the time Q21. FR-I am always thinking about food; Q22. FR-When I see or smell food that I like, it makes me want to eat. (F) Food responsiveness. Q19. FR-I often feel hungry when I am with someone who is eating, Q20. FR-Given the choice, I would eat most of the time Q21. FR-I am always thinking about food; $Q 22$. FR-When I see or smell food that I like, it makes me want to eat.

satiety after eating [24].

With the statement "I am often last at finishing a meal", 58\% (M) and 44\% (F) agreed, which probably could not always be associated with slow food consumption. The results of the survey showed that the speed of food consumption during the meal is decreasing more and more $(24 \%(\mathrm{M})$ and $29 \%(\mathrm{~F})$, and $21 \%(\mathrm{M})$ and 33\% (F) quickly finish eating (Figure 6(M) \& Figure 6(F)).

Fast eating is associated with high BMI and an increased risk of obesity [25].

Hunger. Hunger is supposed to cause overeating, but there is no firm evidence that the intensity of hunger is related to being overweight. $34 \%$ of boys and $45 \%$ of girls often have a strong feeling of hunger that they should eat something immediately. About $14 \%$ of boys and $29 \%$ of girls said they had dizziness due to late meals (Figure 7(M) \& Figure $7(\mathrm{~F})$ ). 


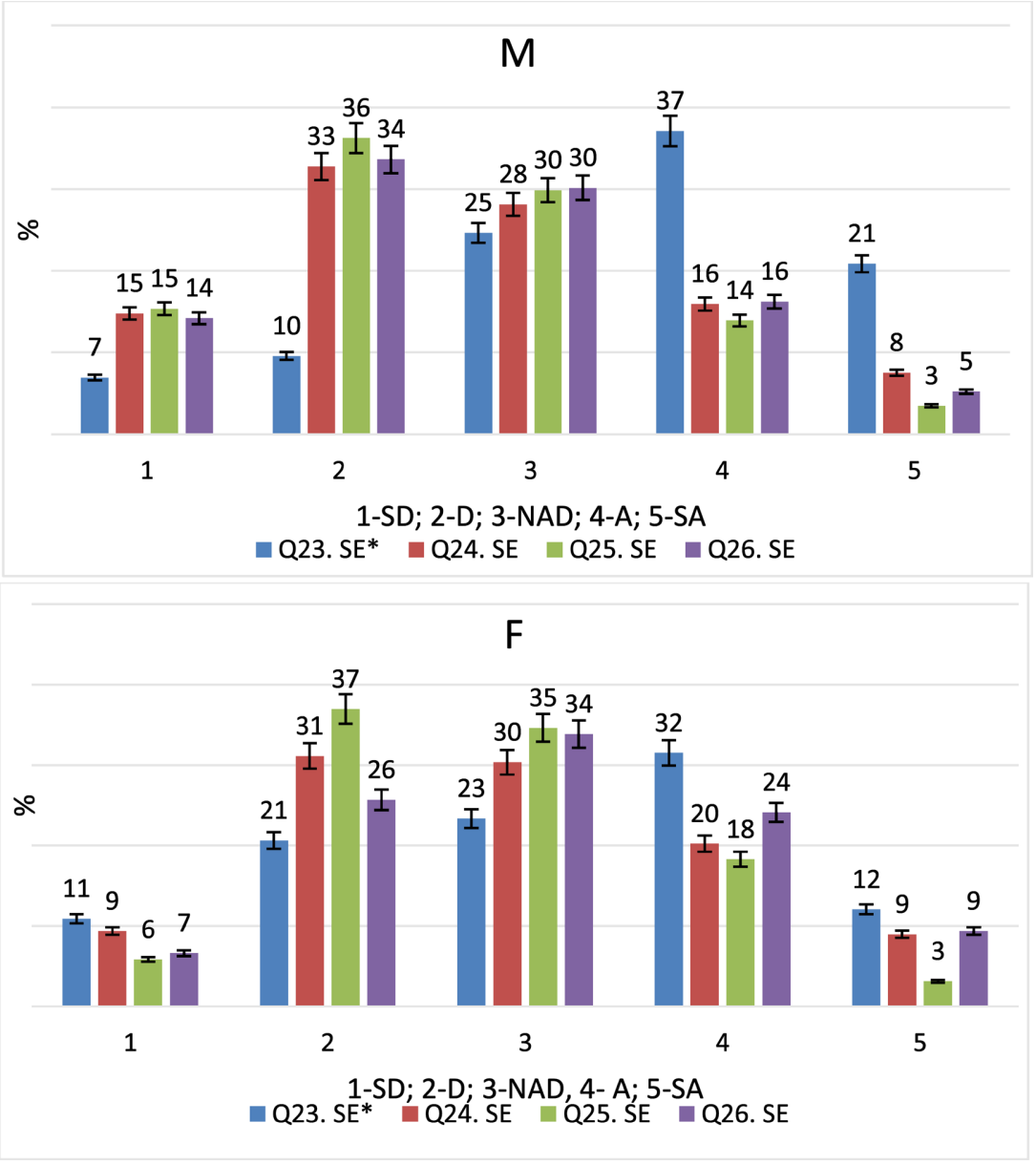

Figure 6. (M) Slowness in eating.Q.23.SE-I am often last at finishing a meal; Q24. SE-I eat more and more slowly during the course of a meal; Q25. SE I eat slowly, Q26. SE*-I often finish my meals quickly (I am often last at finishing a meal). (F) Slowness in eating. Q.23. SE-I am often last at finishing a meal; Q24. SE-I eat more and more slowly during the course of a meal; Q25. SE I eat slowly, Q26. SE*-I often finish my meals quickly ( $I$ am often last at finishing a meal).

Satiety-responsiveness' $(S R)$ examines ability to regulate intake of food in relation to satiety [29]. It is considered that people prone to weaker satiety signals, in response, are more prone to overeating [2]. In this regard, we can conclude that the females reach the feeling of satiety more easily, often leaving food on the plate, which is not characteristic for males. $27 \%$ of boys and $38 \%$ of girls feel full until the end of the meal, and $18 \%$ and $29 \%$ respectively say that they are easily saturated (Figure $8(\mathrm{M})$ \& Figure $8(\mathrm{~F})$ ). Satiety-responsiveness' is a negative association with the quantitative consumption of food, therefore, with higher energy excesses. SR is also associated with the frequency of meals.

\section{Limitations}

The results of the survey were evaluated on a sample of 602 people, which is only a small part of the total number of 9520 TUM students and, respectively, the data obtained may be unrepresentative and may limit the generalization of the data. 


\section{M}
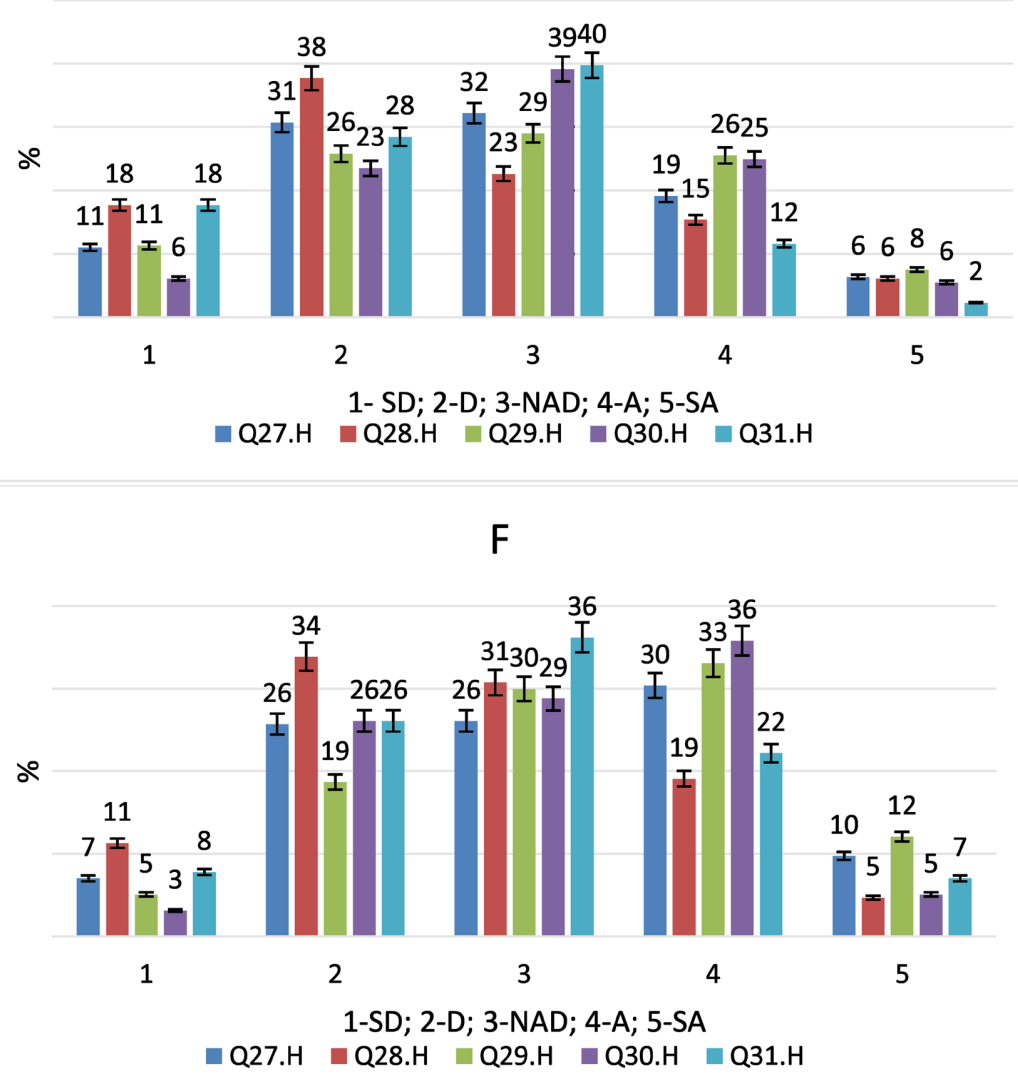

Figure 7. (M) Hunger Sensation.Q27.H-I often notice my stomach rumbling, Q28. H-If I miss a meal I get irritable; Q29. H-I often feel so hungry that I have to eat something right away, Q30. H-I often feel hungry, Q31. H-If my meals are delayed I get lightheaded. (F) Hunger Sensation. Q27. H-I often notice my stomach rumbling, Q28. H-If I miss a meal I get irritable, Q29. H-I often feel so hungry that I have to eat something right away, Q30. H-I often feel hungry, Q31. H-If my meals are delayed I get lightheaded.

An instruction of the respondents for the correct interpretation of the questions would also be suggested. Additional reliable and feasible research on eating behavior, quality of life, and physical activity is needed to improve understanding of the correlations between eating behaviors, energy metabolism, and health.

\section{Conclusions}

Parameters such as Enjoyment of food, Emotional over-eating, Emotional under-eating, Food fussiness, Food responsiveness, Slowness in eating, Hunger, Satiety responsiveness and their gender-dependent oscillations were analyzed. Some relationships have been established between certain emotional states and emotional eating. Emotional states such as nervousness and anxiety are the ones that cause over-eating or under-eating among all students, the girls being the most sensitive to this aspect. Regarding the feeling of hunger, it was associated 


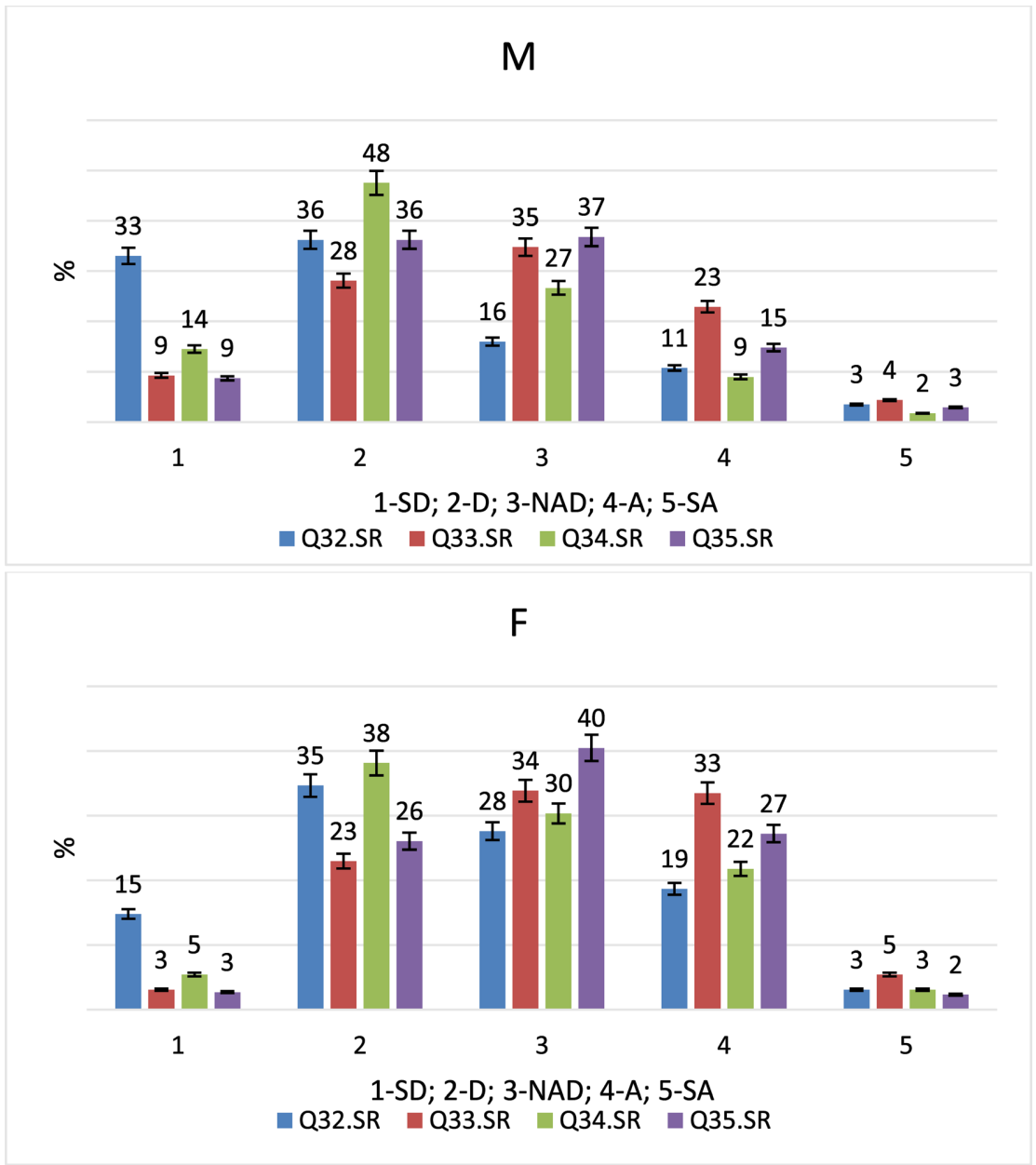

Figure 8. (M) Satiety responsiveness. Q32. SR-I often leave food on my plate at the end of a meal; Q33. SR -I often get full before my meal is finished; Q34. SR-I cannot eat a meal if I have had a snack just before, Q35. SR I I get full up easily. (F) Satiety responsiveness. Q32. SR-I often leave food on my plate at the end of a meal; Q33. SR-I often get full before my meal is finished; Q34.SR - I cannot eat a meal if I have had a snack just before; Q35. SR-I get full up easily.

with the appearance of stomach noises and dizziness and with the parity of irritation. At the same time, girls more easily achieve the feeling of satiety than in the case of boys.

It appears that the results of the AEBQ questionnaire are largely in line with previous studies, and the data collected are of interest, in particular to prevent the risk of obesity. Also, the obtained results could contribute to the formation of the database for the study of the behavioral phenotype associated with the risk of obesity [30], but also for the study of eating behavior in crisis situations.

\section{Funding}

This research was funded by State Project "Personalized nutrition and intelligent technologies for my well-being", grant number 20.80009.5107.10 and Contributions regarding nutritional eradication of gluten consumption dis- 
eases, nr. 21.00208.5107.06/PD, running at Technical University of Moldova (https://utm.md/).

\section{Conflicts of Interest}

The authors declare no conflicts of interest regarding the publication of this paper.

\section{References}

[1] Ashcroft, J., Semmler, C., Carnell, S., et al. (2008) Continuity and Stability of Eating Behaviour Traits in Children. European Journal of Clinical Nutrition, 62, 985-990. https://doi.org/10.1038/sj.ejcn.1602855

[2] Llewellyn, C.H. and Fildes, A. (2017) Behavioural Susceptibility Theory: Professor Jane Wardle and the Role of Appetite in Genetic Risk of Obesity. Current Obesity Reports, 6, 38-45. https://doi.org/10.1007/s13679-017-0247-x

[3] Kvalsvik, F., Øgaard, T. and Jensen, Ø. (2021) Environmental Factors that Impact the Eating Behavior of Home-Living Older Adults. International Journal of Nursing Studies Advances, 3, Article ID: 100046. https://doi.org/10.1016/j.ijnsa.2021.100046

[4] Lim, M.C., Parsons, S., Goglio, A. and Fox, E. (2021) Anxiety, Stress, and Binge Eating Tendencies in Adolescence: A Prospective Approach. Journal of Eating Disorders, 9, Article No. 94. https://doi.org/10.1186/s40337-021-00444-2

[5] Banerjee, D. and Rai, M. (2020) Social Isolation in Covid-19: The Impact of Loneliness. International Journal of Social Psychiatry, 66, 525-527. https://doi.org/10.1177/0020764020922269

[6] Di Renzo, L., Gualtieri, P., Cinelli, G., et al. (2020) Psychological Aspects and Eating Habits during COVID-19 Home Confinement: Results of EHLC-COVID-19 Italian Online Survey. Nutrients, 12, Article No. 2152. https://doi.org/10.3390/nu12072152

[7] Liu, X.H., Kakade, M., Fuller, C.J., et al. (2012) Depression after Exposure to Stressful Events: Lessons Learned from the Severe Acute Respiratory Syndrome Epidemic. Comprehensive Psychiatry, 53, 15-23. https://doi.org/10.1016/j.comppsych.2011.02.003

[8] Grant, F., Scalvedi, M.L., Scognamiglio, U., et al. (2021) Eating Habits during the COVID-19 Lockdown in Italy: The Nutritional and Lifestyle Side Effects of the Pandemic. Nutrients, 13, Article No. 2279. https://doi.org/10.3390/nu13072279

[9] Di Renzo, L., Gualtieri, P., Pivari, F., et al. (2020) Eating Habits and Lifestyle Changes during COVID-19 Lockdown: An Italian Survey. Journal of Translational Medicine, 18, Article No. 229. https://doi.org/10.1186/s12967-020-02399-5

[10] Pellegrini, M., Ponzo, V., Rosato, R., et al. (2020) Changes in Weight and Nutritional Habits in Adults with Obesity during the "Lockdown" Period Caused by the COVID-19 Virus Emergency. Nutrients, 12, Article No. 2016. https://doi.org/10.3390/nu12072016

[11] Scarmozzino, F. and Visioli, F. (2020) Covid-19 and the Subsequent Lockdown Modified Dietary Habits of Almost Half the Population in an Italian Sample. Foods, 9, Article No. 675. https://doi.org/10.3390/foods9050675

[12] Romeo-Arroyo, E., Mora, M. and Vázquez-Araújo, L. (2020) Consumer Behavior in Confinement Times: Food Choice and Cooking Attitudes in Spain. International Journal of Gastronomy and Food Science, 21, Article ID: 100226. https://doi.org/10.1016/j.ijgfs.2020.100226 
[13] Siminiuc, R. and Turcanu, D. (2020) The Impact of the Pandemic on the Agri-Food System. Journal of Social Sciences, 3, 85-94. https://doi.org/10.5281/zenodo.3971973

[14] Hagerman, C.J., Stock, M.L., Beekman, J.B., et al. (2021) The Ironic Effects of Dietary Restraint in Situations that Undermine Self-Regulation. Eating Behaviors, 43, Article ID: 101579. https://doi.org/10.1016/j.eatbeh.2021.101579

[15] Brooks, S.K., Webster, R.K., Smith, L. E., et al. (2020) The Psychological Impact of Quarantine and How to Reduce It: Rapid Review of the Evidence. The Lancet, 395, 912-920. https://doi.org/10.1016/S0140-6736(20)30460-8

[16] Sogari, G., Velez-Argumedo, C., Gómez, M. and Mora, C. (2018) College Students and Eating Habits: A Study Using an Ecological Model for Healthy Behavior. $\mathrm{Nu}$ trients, 10, Article No. 1823. https://doi.org/10.3390/nu10121823

[17] Llewellyn, C. and Wardle, J. (2015) Behavioral Susceptibility to Obesity: Gene-Environment Interplay in the Development of Weight. Physiology \& Behavior, 152, 494-501. https://doi.org/10.1016/j.physbeh.2015.07.006

[18] Hunot-Alexander, C., Beeken, R.J., Goodman, W., et al. (2019) Confirmation of the Factor Structure and Reliability of the 'Adult Eating Behavior Questionnaire' in an Adolescent Sample. Frontiers in Psychology, 10, Article No. 1991.

https://doi.org/10.3389/fpsyg.2019.01991

[19] Țurcanu, D., Siminiuc, R. and Bostan, V. (2020) The Impact of the COVID-19 Pandemic on the Use of Digital Technologies in Ensuring the Efficient e-Learning Process at the Technical University of Moldova. Creative Education, 11, 2116-2132. https://doi.org/10.4236/ce.2020.1110154

[20] Țurcanu, D., Siminiuc, R., Bostan, V. and Țurcanu, T. (2022) Impact of the Covid-19 Pandemic on the Use of Microsoft 365 and Learning Outcomes at the Technical University of Moldova. In: Tiginyanu, I., Sontea, V. and Railean, S., Eds., 5 th International Conference on Nanotechnologies and Biomedical Engineering, Chisinau, 3-5 November 2021, 456-462.

[21] Hunot, C., Fildes, A., Croker, H., et al. (2016) Appetitive Traits and Relationships with BMI in Adults: Development of the Adult Eating Behaviour Questionnaire. Appetite, 105, 356-363. https://doi.org/10.1016/j.appet.2016.05.024

[22] Wiggins, S. (2019) Moments of Pleasure: A Preliminary Classification of Gustatory mmms and the Enactment of Enjoyment during Infant Mealtimes. Frontiers in Psychology, 10, Article No. 1404. https://doi.org/10.3389/fpsyg.2019.01404

[23] Herle, M., Fildes, A., Steinsbekk, S., et al. (2017) Emotional Over- and Under-Eating in Early Childhood Are Learned Not Inherited. Scientific Reports, 7, Article No. 9092. https://doi.org/10.1038/s41598-017-09519-0

[24] Macht, M. (2008) How Emotions Affect Eating: A Five-Way Model. Appetite, 50, 1-11. https://doi.org/10.1016/j.appet.2007.07.002

[25] Hunot-Alexander, C., Arellano-Gómez, L.P., Smith, A.D., et al. (2021) Examining the Validity and Consistency of the Adult Eating Behaviour Questionnaire-Español (AEBQ-Esp) and Its Relationship to BMI in a Mexican Population. Eating and Weight Disorders-Studies on Anorexia, Bulimia and Obesity. https://doi.org/10.1007/s40519-021-01201-9

[26] Gibson, E.L. and Cooke, L. (2017) Understanding Food Fussiness and Its Implications for Food Choice, Health, Weight and Interventions in Young Children: The Impact of Professor Jane Wardle. Current Obesity Reports, 6, 46-56. https://doi.org/10.1007/s13679-017-0248-9

[27] Ervina, E., Almli, V.L., Berget, I., et al. (2021) Does Responsiveness to Basic Tastes Influence Preadolescents' Food Liking? Investigating Taste Responsiveness Segment 
on Bitter-Sour-Sweet and Salty-Umami Model Food Samples. Nutrients, 13, Article No. 2721. https://doi.org/10.3390/nu13082721

[28] Schachter, S. (1968) Obesity and Eating: Internal and External Cues Differentially Affect the Eating Behavior of Obese and Normal Subjects. Science, 161, 751-756. https://doi.org/10.1126/science.161.3843.751

[29] Brown, A. and Lee, M.D. (2015) Early Influences on Child Satiety-Responsiveness: The Role of Weaning Style. Pediatric Obesity, 10, 57-66. https://doi.org/10.1111/j.2047-6310.2013.00207.x

[30] Carnell, S. and Wardle, J. (2007) Measuring Behavioural Susceptibility to Obesity: Validation of the Child Eating Behaviour Questionnaire. Appetite, 48, 104-113. https://doi.org/10.1016/j.appet.2006.07.075 


\section{Appendix 1. Adult Eating Behaviour Questionnaire-Scoring Information}

\begin{tabular}{|c|c|c|c|c|c|c|}
\hline n.o. & & $\begin{array}{l}\text { Strongly } \\
\text { disagree }\end{array}$ & Disagree & $\begin{array}{l}\text { Neither } \\
\text { agree or } \\
\text { disagree }\end{array}$ & Agree & $\begin{array}{c}\text { Strongly } \\
\text { agree }\end{array}$ \\
\hline & Enjoyment of food & 1 & 2 & 3 & 4 & 5 \\
\hline Q1. EF & I love food & & & & & \\
\hline Q.2. EF & I enjoy eating & & & & & \\
\hline \multirow[t]{2}{*}{ Q.3. EF } & I look forward to mealtimes & & & & & \\
\hline & Emotional over-eating & & & & & \\
\hline Q.4. EOE & I eat more when I'm annoyed & & & & & \\
\hline Q.5. EOE & I eat more when I'm worried & & & & & \\
\hline Q.6. EOE & I eat more when I'm upset & & & & & \\
\hline Q.7. EOE & I eat more when $I^{\prime} m$ anxious & & & & & \\
\hline \multirow[t]{2}{*}{ Q.8. EOE } & I eat more when I'm angry & & & & & \\
\hline & Emotional under-eating & & & & & \\
\hline Q.9. EUE & I eat less when I'm worried & & & & & \\
\hline Q.10. EUE & I eat less when I'm angry & & & & & \\
\hline Q.11. EUE & I eat less when I'm upset & & & & & \\
\hline Q.12. EUE & I eat less when I'm annoyed & & & & & \\
\hline \multirow[t]{2}{*}{ Q.13. EUE } & I eat less when I'm anxious & & & & & \\
\hline & Food fussiness & & & & & \\
\hline Q.14. FF & I often decide that I don't like a food, before tasting it & & & & & \\
\hline Q.15. FF & I refuse new foods at first & & & & & \\
\hline Q.16. $\mathrm{FF}^{\star}$ & I enjoy tasting new foods & & & & & \\
\hline Q.17. $\mathrm{FF}^{*}$ & I am interested in tasting new food I haven't tasted before & & & & & \\
\hline \multirow[t]{2}{*}{ Q.18. $\mathrm{FF}^{*}$} & I enjoy a wide variety of foods & & & & & \\
\hline & Food responsiveness & & & & & \\
\hline Q.19. FR & I often feel hungry when I am with someone who is eating & & & & & \\
\hline Q.20. FR & Given the choice, I would eat most of the time & & & & & \\
\hline Q.21. FR & I am always thinking about food & & & & & \\
\hline \multirow[t]{2}{*}{ Q.22. FR } & When I see or smell food that I like, it makes me want to eat & & & & & \\
\hline & Slowness in eating & & & & & \\
\hline Q.23. SE* & I am often last at finishing a meal & & & & & \\
\hline Q.24. SE & I eat more and more slowly during the course of a meal & & & & & \\
\hline Q.25. SE & I eat slowly & & & & & \\
\hline Q.26. SE & I often finish my meals quickly & & & & & \\
\hline
\end{tabular}




\section{Continued}

\section{Hunger}

Q.27. H I often notice my stomach rumbling

Q.28. H If I miss a meal I get irritable

Q.29. H I often feel so hungry that I have to eat something right away

Q.30. H I often feel hungry

Q.31. H If my meals are delayed I get lightheaded

\section{Satiety responsiveness}

Q.32.SR I often leave food on my plate at the end of a meal

Q.33. SR I often get full before my meal is finished

Q.34. SR I cannot eat a meal if I have had a snack just before

Q.35. SR I get full up easily 